\title{
Mild-intensity Exercise Triggers VEGF in the Digestive Tract Via Both Hypoxic and Nonhypoxic Mechanisms
}

\author{
Ferda Hosgorler ${ }^{1^{*}}$ \\ http://orcid.org/0000-0001-6846-5312
}

Servet Kizildag ${ }^{2}$

http://orcid.org/0000-0003-3565-279X

Basar Koc ${ }^{1}$

http://orcid.org/0000-0003-2621-1693

\section{Oguz Yüksel ${ }^{3}$}

https://orcid.org/0000-0003-1834-0444

Ayse Birsu Topcugil Kırık

https://orcid.org/0000-0002-6296-9760

\author{
Rabia llgin 1 \\ https://orcid.org/0000-0002-9759-7485
}

Sevim Kandis ${ }^{1}$

http://orcid.org/0000-0001-8992-7985

Güven Güvendi ${ }^{1}$

http://orcid.org/0000-0002-1858-162X

Mehmet Ates ${ }^{2}$

http://orcid.org/0000-0002-8310-1979

Nazan Uysal ${ }^{1}$

http://orcid.org/0000-0002-2348-7427

1'Dokuz Eylül University, School of Medicine, Department of Physiology, Izmir, Turkey; '2Dokuz Eylül University, College of Vocational School of Health Services, Izmir, Turkey; ${ }^{3}$ Department of Sports Medicine, Dokuz Eylül University, School of Medicine, Izmir, Turkey.

Received: 2020.02.02; Accepted: 2020.04.24.

*Correspondence: ferda.hosgorler@deu.edu.tr; Tel.: +905074831184/+902324122222

HIGHLIGHTS

- Low vascular endothelial growth factor expression is observed in the elderly.

- Exercise leads to hypoxia in the splanchnic region.

- Mild intensity exercise induces vascular endothelial growth factor in the elderly.

- In older subjects, mild intensity exercise increases antioxidant capacity in the stomach.

- Hypoxic involvement due to exercise is more pronounced in the large intestine compared to other parts of the digestive tract.

Abstract: Hypoxia occurs in the splanchnic region during exercise associated with sympathetic activity. In the elderly, vascular insufficiency and low vascular endothelial growth factor (VEGF) expression are observed. Compared to young people, sympathetic signals of older individuals are blunted and more resistant to splanchnic blood flow alterations during exercise. VEGF induces vasodilation responses and hence may retain blood in the splanchnic vascular bed. We hypothesized that regular mild-intensity exercise triggers weak VEGF expression in the digestive tract of the elderly. The effects of exercise on the levels of VEGF, superoxide dismutase (SOD), glutathione peroxidase (GPx), malondialdehyde (MDA) and total antioxidant capacity (T-AOC) in the stomach, jejunum, ileum and colon tissues were evaluated. With exercise, the VEGF levels in the stomach and colon increased. Although the SOD, GPx, and MDA levels decreased in the 
stomach, they increased in the colon. T-AOC increased in the stomach and there was no change in the jejunum, ileum and colon. The hypoperfusion during exercise was not equal in all regions of the gastrointestinal tract in the aged subjects. Hypoxia and other exercise-related mechanisms could have led to this VEGF induction. The stomach, jejunum, and ileum might have developed resistance to ischemia. The induction of VEGF may be beneficial in aging-associated impaired gastrointestinal homeostasis and neovascularization.

Keywords: exercise; gastrointestinal; hypoxia; VEGF.

\section{INTRODUCTION}

Adequate blood flow to the gastrointestinal tract is essential for the supply of nutrients and oxygen and the removal of toxic metabolic products. In the gastrointestinal tract, a reduction in blood flow up to $75 \%$ during and after exercise related with increased sympathetic activity and gastrointestinal complaints, has been reported [1,2]. The degree of ischemia is associated with exercise intensity. Severe and prolonged exercise can lead to endothelial dysfunction and enterocyte injury [3,4]. However, mild and moderate exercise effects on gastrointestinal tractus are more slight, and may yield beneficial effects such as decreasing gastric secretion and increasing immune resistance [3,5].

Gastrointestinal symptoms and diseases gradually increase with advancing age [6]. Symptoms arising from structural deterioration of the digestive canal wall, impaired motility function, and decreased blood flow may occur in the elderly [7]. Mesenteric vasculopathy (such as endothelial dysfunction, atherosclerosis) has been reported in more than $50 \%$ of the elderly [8]. As a result of vasculopathy, the digestive system of the elderly may be more sensitive to the decrease in splanchnic blood flow during exercise. Studies have indicated that the splanchnic blood flow in the elderly during moderate-intensity exercise is less impaired than in the young [9]. The effects of mild exercise and the possible impacts in vasculopathy in the digestive systems of the elderly are not clear.

VEGF induces the growth of pre-existing vessels (angiogenesis) or de novo vessels (vasculogenesis) to provide adequate oxygen to the tissues [10]. VEGF over-expression in the postnatal murine duodenum was found to lead to an increase in mucosal angiogenesis and vascular permeability, in addition to a rise in villus height and the enhancement in the proliferation of epithelial cells [11]. The importance of VEGF in the gastrointestinal tract has been highlighted in the pathogenesis of necrotizing enterocolitis (NEC) and intestinal complications (such as edema, hemorrhage, perforation) of anti-VEGF treatments [12,13].

Research has shown that the VEGF expression in human dermal microvascular endothelial cells (HMVECs) and gastric mucosal endothelial cells derived from elderly individuals had decreased [14]. Due to its role in endothelial cell function, new vessel formation and in the repair processes, VEGF expression is critical in gastrointestinal homeostasis. The expressions of VEGF are regulated by hypoxia and the oxidants at the transcriptional level [15]. VEGF has been known to be triggered by Hypoxia-inducible factor-1 alpha (HIF-1 $\alpha$ ) and Reactive oxygen species (ROS) [16]. HIF-1 $\alpha$ regulates VEGF gene expression and provides the adaptation responses necessary for the maintenance of gastrointestinal homeostasis in hypoxia [17]. When various cells are exposed to superoxide or hydrogen peroxide in vivo and in vitro, VEGF mRNA expressions were elevated [18]. VEGF can also be stimulated by exercise with the stimulation of lactate receptors without the occurrence of hypoxia [19]. Increases in VEGF expression in the blood have also been observed in some elderly exercise studies [20]. It was reported that VEGF expression was stimulated in striated muscle by mild exercise [21]. These results support the tenet that mild exercise may be effective in triggering VEGF in the gastrointestinal tracts of elderly subjects by hypoxic or non-hypoxic mechanisms.

Elderly individuals are prone to mucosal chronic infection (inflamm-aging) and the development of mucosal lesions. The healing of these pathologies is not only difficult, but the functions of the endothelial cells are also impaired and their mucosal microcirculation is poor $[22,23]$. Changes in microbiota caused by advancing age disrupt immune homeostasis [24]. The role of mucosal blood flow is fundamental in the development or recovery of many age-related increased gastrointestinal diseases such as gastric ulcers, atrophic gastritis, mucosal dysplasia, mesenteric ischemia, diverticular disease, inflammatory bowel disease, and colorectal cancer [25]. Literature supports the positive effects of low-intensity exercise on the physical and cognitive health of the elderly [26]. However, the effects of low-intensity exercise on the blood flow in the digestive tracts should be further investigated. Consequently, the question to be considered is whether regular low-intensity exercise should be recommended to the elderly, especially those with digestive complaints. 
We hypothesized that in elderly subjects, regular aerobic mild-intensity exercise would stimulate VEGF responses in the digestive tract. The aim of the study is to evaluate the changes in VEGF expressions in the gastrointestinal tract in mild exercise, and the relationship of this change as regards redox status.

\section{MATERIAL AND METHODS}

The tissue materials that were used in the current protocol were obtained from another protocol: The Institutional Review Board/Ethical Review Board No. 48-2018 entitled "Investigation of the effects of regular aerobic exercise on cognitive function in elderly rats". The study had been previously approved by the institutional ethics committee. All experimental procedures were performed by the experimental study team in accordance with the Guide for the Care and Use of Laboratory Animals.

Old male Sprague Dawley rats aged 22-23 months were housed in individual cages with free access to water and laboratory chow (general animal data are shown in the Table 1). Standard rodent chow was provided in oval pellet form (containing crude protein at least $23.0 \%$, crude fiber no more than $7.0 \%$, crude fat at least $3.0 \%, 265 \mathrm{kcal} / 100 \mathrm{gr}$ ). The animals were kept in a $12 \mathrm{~h}$ light-12 h dark cycle. Room temperature $\left(22^{\circ} \pm 1^{\circ} \mathrm{C}\right)$ and humidity $(60 \%)$ were kept constant. One week of handling was performed to enable the rats to adapt to the experimental environment.

Table 1. General animal data of the animals (The values are presented with mean and standard deviation)

\begin{tabular}{lllll}
\hline & $\begin{array}{l}\text { Body weight } \\
\text { before } \\
\text { experiment (g) }\end{array}$ & $\begin{array}{l}\text { Body weight at the } \\
\text { end of the 6th week }(\mathbf{g})\end{array}$ & $\begin{array}{l}\text { Average chow } \\
\text { intake (rat/day) }(\mathbf{g})\end{array}$ & $\begin{array}{l}\text { Average water intake } \\
\text { (rat/day) }(\mathbf{m L})\end{array}$ \\
\hline Exercise group & $470 \pm 32$ & $435 \pm 30$ & $72 \pm 22$ & $70 \pm 10$ \\
\hline Control group & $442 \pm 41$ & $437 \pm 48$ & $65 \pm 14$ & $65 \pm 15$ \\
\hline
\end{tabular}

\section{Experimental Design}

The experiments were conducted in the institutional Physiology Laboratory between 08:00 to 12:00 a.m. daily. The rats were divided into two groups: (1) exercise group $(n=7)$ and (2) control group $(n=7)$.

During exercise, the treadmill device for rodents (May TME 0804 Animal Treadmill, Commant Ltd, Ankara, Turkey), on which speed and slope can be adjusted to enable the animals to walk on the rolling belts, was used. The exercise group underwent 1 week of treadmill training to learn and adapt at $5 \mathrm{~m} / \mathrm{min} 0^{\circ}$ slope, $10 \mathrm{~min} /$ day, 5 days/week. The rats were then trained on the treadmill 30 minutes/day, 3 days/week at a speed of $8 \mathrm{~m} / \mathrm{min}$ with mild exercise intensity for 6 weeks. This exercise protocol is considered mild intensity for elderly rats [27]. We selected a mild exercise intensity protocol which can be regularly implemented by the elderly and may yield beneficial effects (such as providing immune support and gastric acid regulation) [3,5]. The control group was brought to the experimental room and subjected to identical handling. All the analyses were performed for both groups.

Two days after the completion of the exercise phase, the rats were sacrificed under $\mathrm{CO}_{2}$ inhalation anesthesia, which has a rapid and short-term anesthetic effect [28]. The animals were euthanized by decapitation. Tissue samples were obtained from the distal sections of the stomach antrum, proximal ileum segment, proximal jejunum segment, and distal sigmoid loops of the large intestine. Tissue samples were harvested on the ice ground and placed in the refrigerator $\left(-80^{\circ} \mathrm{C}\right)$ immediately after collecting.

\section{Biochemical Measurements}

The gastrointestinal tissues were weighed and homogenized with steel beads by using a BioSpec MiniBeadbeater-16 (BioSpec Products Inc., Bartlesville, OK, USA) in 10 volumes of Phosphate-buffered saline (PBS) $(\mathrm{pH}=7.4)$. The tissue homogenates were centrifuged at $5,000 \mathrm{~g}$ for $15 \mathrm{~min}$ at $4{ }^{\circ} \mathrm{C}$ in refrigerated centrifuge. The supernatants were used for all biochemical analyses.

Tissue enzyme-linked immunosorbent assay (ELISA) measurements for VEGF, SOD, GPx, and MDA were performed in accordance with the kit protocol. The VEGF levels were measured with commercially available rat-specific ELISA kits (VEGF Catalog No. EK0540, Boster Immunoleader, Pleasanton, USA, with an assay sensitivity of $<1 \mathrm{pg} / \mathrm{mL}$ and range of $15.6-1000 \mathrm{pg} / \mathrm{mL}$ ). Rat-specific SOD kit (Catalog No. E0168Ra, Bioassay Technology Laboratory, Shanghai, China, with an assay sensitivity of $<0.022 \mathrm{ng} / \mathrm{mL}$ and detection range of $0.05-20 \mathrm{ng} / \mathrm{mL}$ ) were also used in addition to a rat-specific GPx kit (Catalog No. E1242Ra, Bioassay Technology Laboratory, Shanghai, China, with an assay sensitivity of $<0.24 \mathrm{ng} / \mathrm{mL}$ and detection range of 0.5-200 ng/mL), and a rat-specific MDA kit (Catalog No. E0156Ra, Bioassay Technology Laboratory, Shanghai, China, with an assay sensitivity of $<0.01 \mathrm{nmol} / \mathrm{mL}$ and detection range of $0.05-10 \mathrm{nmol} / \mathrm{mL}$ ). All 
results were expressed as per mg of tissue protein. Protein analysis was performed in accordance with the manufacturer's guidelines for the BCA protein assay kit (Catalog No. 23227, Pierce ${ }^{\mathrm{TM}}$ BCA Protein Assay Kit, Thermo Fisher Scientific, Waltham, MA, USA). The absorbency changes were measured with a microplate reader (ELx800, BioTek Instruments, Inc., Winooski, VT, USA) at $450 \mathrm{~nm}$ for the ELISA kits and $560 \mathrm{~nm}$ for the protein assay kit.

Total Antioxidant Capacity (T-AOC) Assay Kit (FRAP method) (Catalog No: E-BC-K225-M, Elabscience, Wuhan, China) were used in accordance with the kit protocol. In this method principle of detection is that $\mathrm{Fe}^{3+}-2,4,6$-tripyridyl-s-triazine (TPTZ) complex can be reduced by antioxidants and produce blue $\mathrm{Fe}^{2+}-\mathrm{TPTZ}$ under acid condition. The antioxidant capacity of sample can be calculated by detection the absorbance value at $590-600 \mathrm{~nm}$. The absorbency changes were measured with a microplate reader (Synergy HT, BioTek Instruments, Winooski, VT, USA) at $595 \mathrm{~nm}$ for T-AOC kit.

\section{Statistical Analysis}

Statistical analyses were performed with IBM SPSS Statistics for Windows, Version 22.0 (IBM, Turkey). The Mann-Whitney $U$ test was used to analyze the statistical significance of the differences between the groups. The correlations between the chemical parameters were calculated with Spearman's rank correlation coefficients. The results are presented as median, minimum, maximum values and percentiles; $p$ values of $<0.05$ were considered statistically significant.

\section{RESULTS}

The comparison with the control group indicated that in both the stomach and the colon, the VEGF levels had increased in the exercise group (for the stomach $p=0.0017$; for the colon $p=0.0017$ [Fig. 1]). There was no difference in the VEGF levels in the jejunum and ileum tissues $(p>0.05$; Fig. 1$)$ related to exercise.

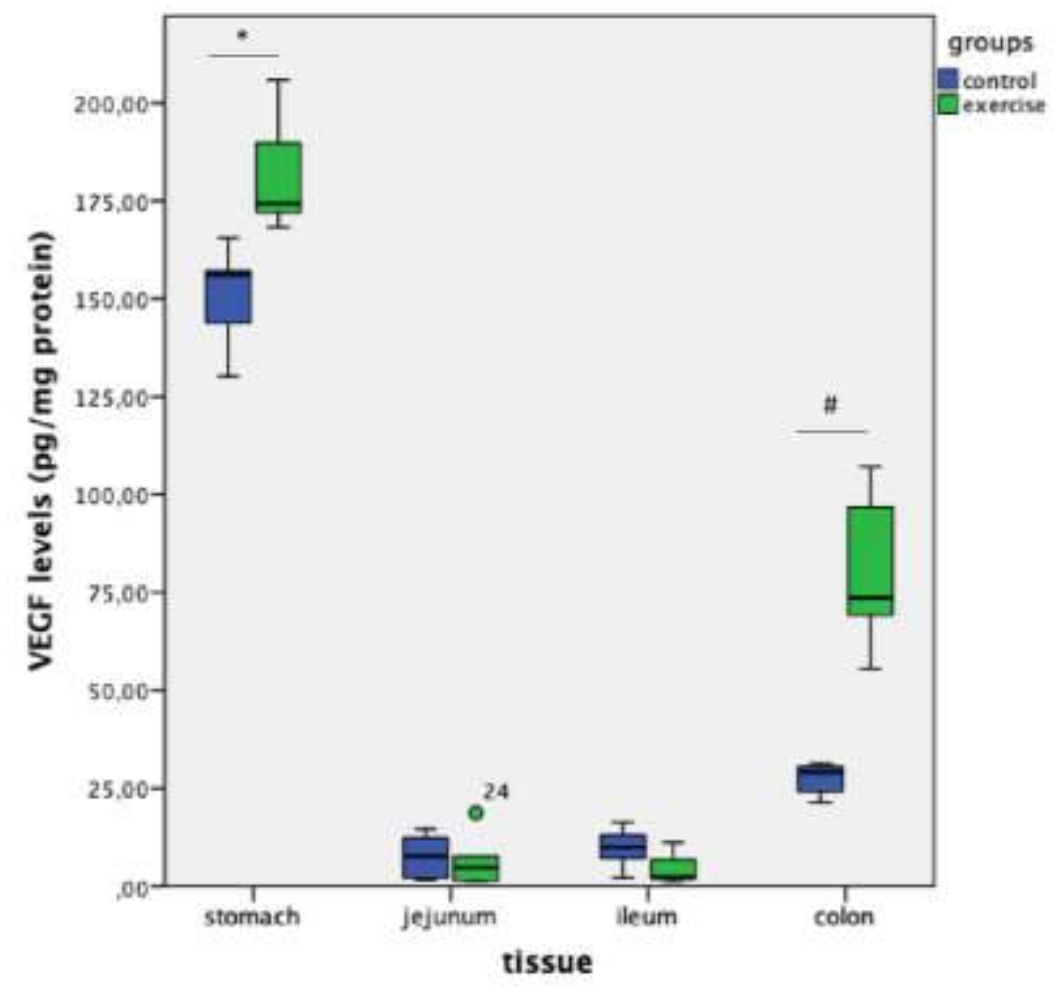

Figure 1. Vascular endothelial growth factor (VEGF) levels in the gastrointestinal tract. Compared to the control group in the exercising group, VEGF levels were higher in the stomach and colon $\left({ }^{*} p<0.01, \# p<0.01\right)$. 
In the exercise group, the SOD levels in the stomach were significantly lower than those in the control group. However, they increased in the colon ( $p=0.0016$ for the stomach, $p=0.006$ for the colon [Figure 2A, 2-B]). Similarly, the comparison with the control group indicated that in the exercise group, the GPx and MDA levels had decreased in the gastric tissues; however, they were elevated in the colon tissues (for GPx in the stomach, $p=0.0017$; for MDA in the stomach, $p=0.0017$; for GPx in the colon, $p=0.012$; and for MDA in the colon, $p=0.0026$ [Figure 2-A, 2-B]). There was no difference between groups in terms of the MDA and antioxidant enzyme levels in the jejunum and ileum tissues (Fig. 2-C, 2-D).
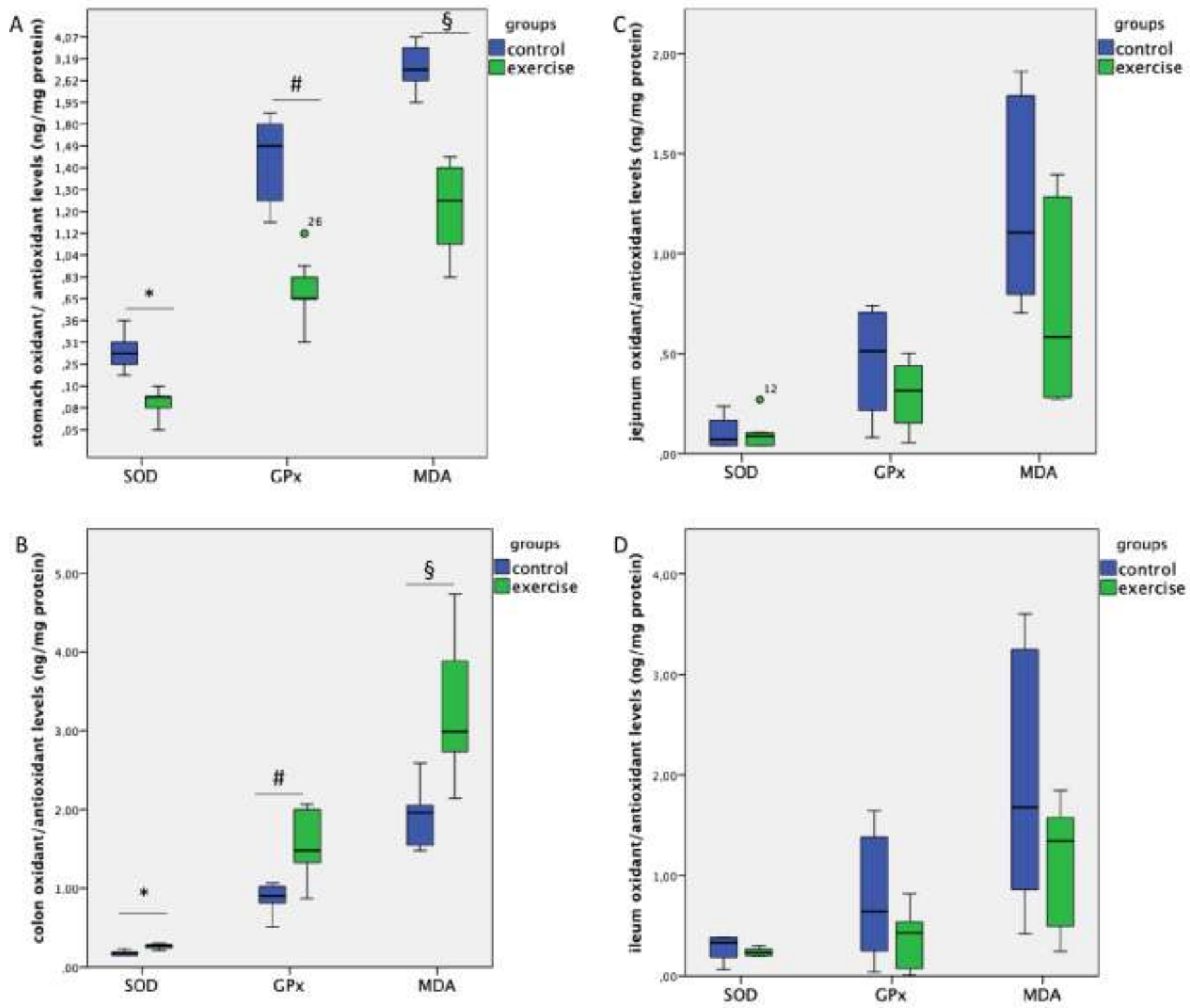

Figure 2. Malondialdehyde (MDA) and Superoxide dismutase (SOD), Glutathione Peroxidase (GPx) enzyme levels in gastrointestinal tract. A. The comparison with the control group indicated that the SOD, GPx, MDA enzyme levels were significantly lower in the stomach tissues of the exercise group $(*, \#, \delta \mathrm{p}<0.01)$. B. The SOD, GPx and MDA levels of colonic tissues in the exercise group were higher than those in the colonic tissues of the control group $\left({ }^{*} p<0.01\right.$, $\# p<0.05, \S p<0,01)$. C and D. MDA and antioxidant enzyme levels were similar in groups jejunum and ileum tissues $(p>0.05)$. 
T-AOC was significantly increased in the stomach and unchanged in the jejunum and ileum $(p=0.003$ for the stomach; Fig.3). Although T-AOC decreased in the colon, this difference was not significant $(p=$ 0.055).

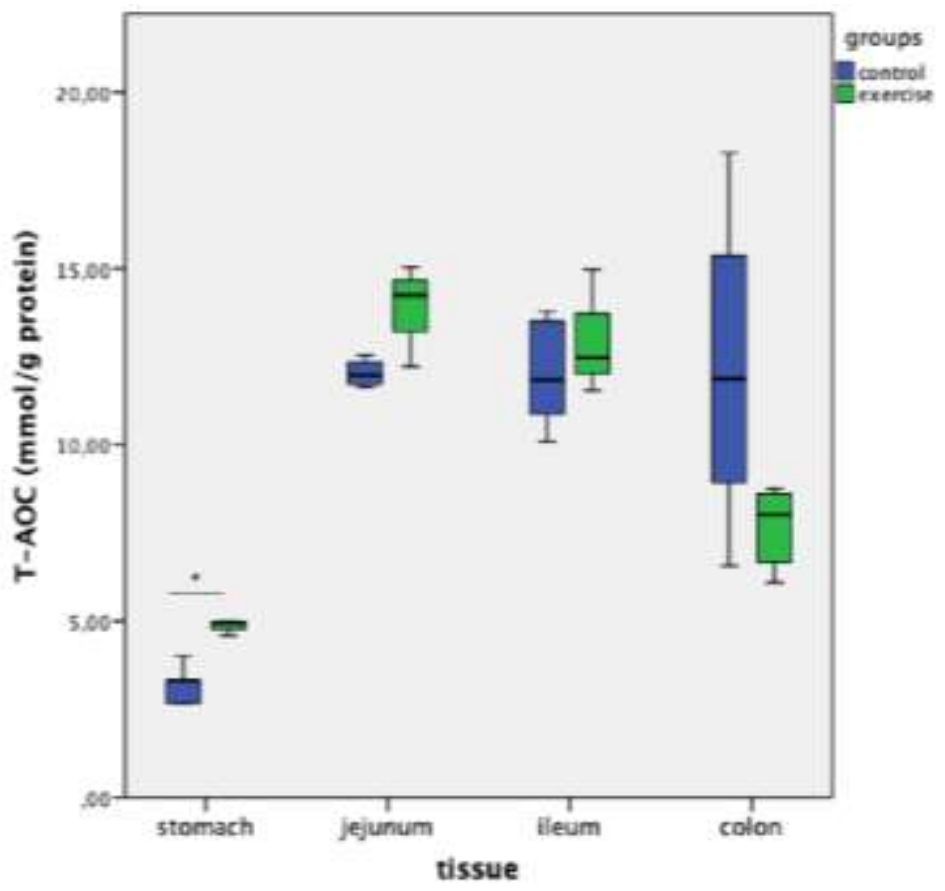

Figure 3. Total antioxidant capacity (T-AOC) in the stomach, jejunum, ileum and colon tissues. T-AOC measurements were higher in the stomach tissues of the exercise group $\left({ }^{*} p<0.01\right)$.

The VEGF levels in the stomach did not exhibit correlations with the SOD, GPx, and MDA levels. However, the VEGF levels highlighted a strong positive correlation with the SOD, GPx, and MDA levels in the colon $(r=0.800, p=0.002$ for SOD; $r=0.736, p=0.003$ for GPx; and $r=0.855, p<0.0001$ for MDA, respectively, as indicated in Fig. 4-B). The MDA levels were strongly correlated with the SOD and GPx in both the stomach and the colon (for the stomach, $r=0.812, p<0.0001$ related to SOD; $r=0.893, p<0.0001$ related to GPx, for the colon, $r=0.800, p=0.002$ regarding SOD and $r=0.732, p=0.003$ regarding GPx respectively).
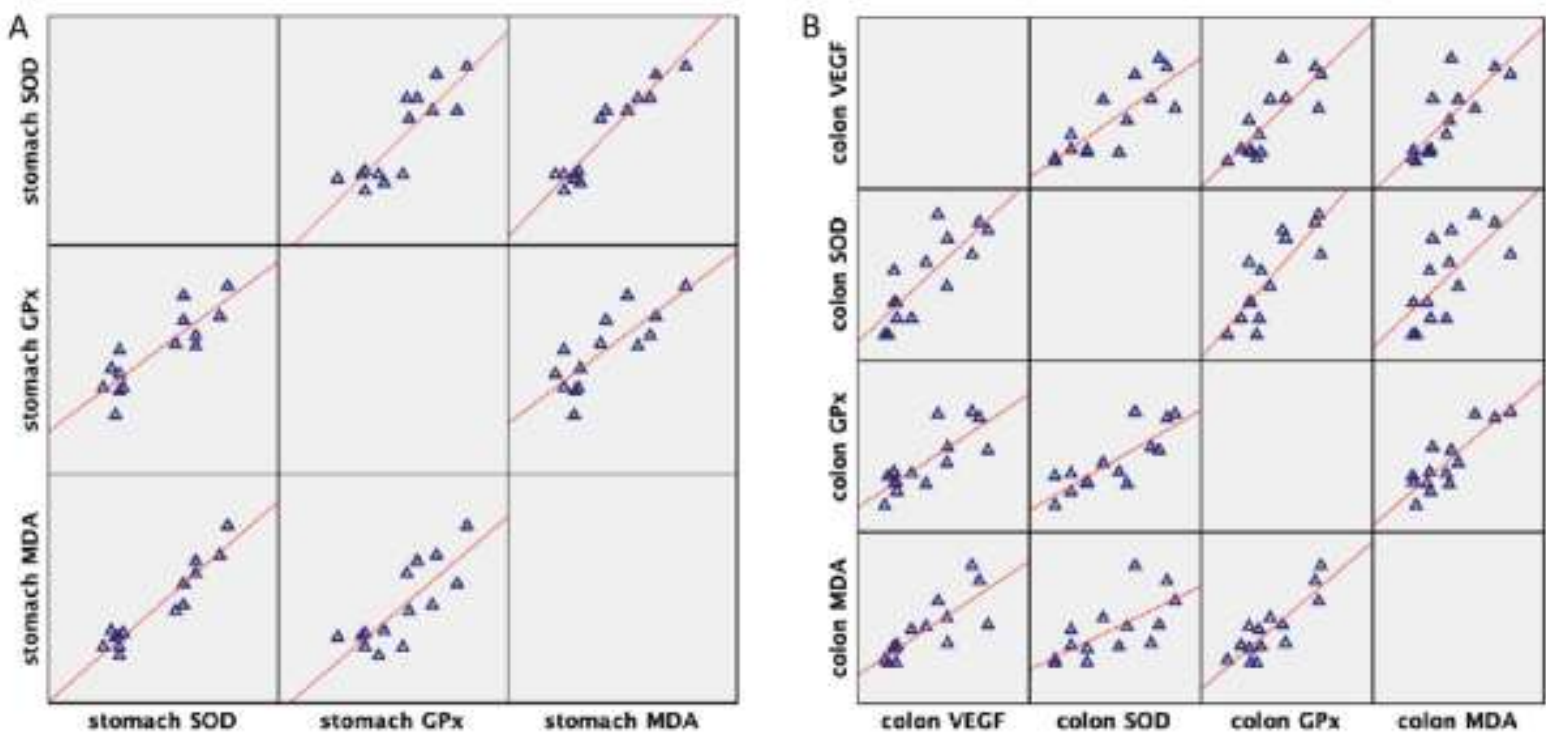

Figure 4. Correlation analysis of biochemical values in the stomach and colon. A. MDA levels exhibited a positive correlation with both the superoxide dismutase (SOD) and the glutathione peroxidase (GPx) enzyme levels $(p<0.0001$ for SOD and GPx) in the stomach tissues. B. The vascular endothelial growth factor (VEGF) levels exhibited a positive correlation with the oxidative markers $(p<0.01$ for SOD, GPx and $p<0.0001$ MDA). The malondialdehyde (MDA) exhibited a positive correlation with both the superoxide dismutase (SOD) and the glutathione peroxidase (GPx) $(p<0.01$ for SOD and GPx). 


\section{DISCUSSION}

In this elderly rat study, regular low-intensity aerobic exercise increased the VEGF levels in the stomach and colon. In addition, there was a shift towards the anti-oxidation state in the stomach and to an oxidation state in the sigmoid colon as regards redox balance.

The circulation in the gastrointestinal tract is normally responsible for $25 \%$ of the total resting cardiac output and total body oxygen consumption [29]. This amount is reduced by the redistribution of cardiac output, and the directing of the blood flow to the muscle during exercise [1]. The severity of the reduction in blood flow to the splanchnic region is influenced by several factors, such as exercise intensity, fasting status, weight, and age [30]. Compared to younger people, the decrease in splanchnic blood flow during exercise is less pronounced in the elderly [9]. The sympathetic responses of the elderly to the redistribution of blood flow to the skeletal muscles were diminished. Therefore, the decrease in blood flow to the splanchnic region during exercise is affected less [31]. The induction of VEGF associated with exercise we detected, may contribute to the retention of blood in the splanchnic region because of the vasodilator effect. VEGF expression yield vasorelaxation responses through nitric oxide (NO) production, decreases blood pressure and regulates the bloodstream [32-34]. Vasodilation effects of VEGF on the digestive tract of old rats during exercise might contribute in the preservation of blood circulation to this region.

We detected a tendency of anti-oxidation in redox status in the stomach with exercise. This situation following exercise may demonstrate that blood flow did not decrease in this region, nor was the stomach affected by hypoxia. The stomach is an organ which possesses strong adaptation mechanisms against stress stimuli. The exposure of the stomach to recurrent mild stressors can engender protective-curative responses, i.e., "adaptive cytoprotection" [35]. The prostaglandins, vasoactive mediators, and nitric oxide can produce adaptive responses by regulating blood flow and supporting mucosal stability in the stomach [36]. Repetitive ischemia stress may create adaptation responses. There is no evidence of impaired adaptive cytoprotecting responses due to advanced age [37]. Studies have shown the production of $\mathrm{HIF-1 \alpha}$ and $\mathrm{ROS}\left(\mathrm{O}_{2}{ }^{-}, \mathrm{H}_{2} \mathrm{O}_{2}\right.$, $\mathrm{HOCl}$ ) triggered in gastric ischemia-reperfusion injuries have been prevented with repeated ischemia. In addition, apoptosis, lipid peroxidation, and post-ischemic oxidative damage were alleviated [38,39]. In our present study, it is possible the recurrent exercise-induced hypoxia for the 6-week duration promoted the development of tolerance to ischemia and diminished ROS production. MDA, the product of lipid peroxidation, and antioxidant enzyme levels decreased following exercise in the stomach. In a similar study, the effectiveness of exercise in reducing the expression of the lipid peroxidation product Thiobarbituric Acid Reactive Substances (TBARS) was demonstrated [40]. MDA production might be associated with the decreased ROS created by the gastric mucosal epithelium, neutrophil, or other cellular sources. It is well known that in a healthy body, there is a certain balance between free radicals and antioxidants. When the free radicals are increased, antioxidant production is triggered, and the free radicals are removed [41]. The reduction in free radicals may also lead to the suppression of intracellular enzymatic antioxidants. We recorded the decreased levels of SOD and GPx in parallel with the MDA levels in the stomach and a positive correlation between the two. T-AOC levels measured for determination of antioxidant status in the stomach tissue increased in the exercise group. T-AOC is known to indicate the effectiveness of all intracellular, extracellular, enzymatic and nonenzymatic antioxidants. While intracellular enzymatic antioxidants decreased, the levels of total antioxidants increased with exercise. It is reported that the increases in SOD and GPx levels are indicative of some disease progression (such as autoimmune skin-mucous membrane disease and inflammatory bowel disease) associated with oxidative stress [42,43]. In these diseases, SOD and GPx levels and TAOC levels have been shown to be inversely related $[42,44]$. Our results regarding mild exercise demonstrated that there was no pronounced hypoxia in the stomach nor a shift in the balance in the oxidative status in the antioxidant direction. Since hypoxia markers do not increase in the stomach, VEGF expression should be stimulated in the stomach by mechanisms independent of hypoxia. Exercise can yield VEGF stimulating effects in older people [20]. Peroxisome proliferator-activated receptor- $\gamma$ coactivator $1 \alpha$ (PGC-1 $\alpha$ ), known as the modulator of mitochondrial biogenesis, and other possible mechanisms (such as lactate) could provoke VEGF $[19,45]$. Research has indicated that through exercise, there has been increased PGC-1 $\alpha$ expression and mitochondrial biogenesis, and that mitochondrial functions have improved in the elderly [46]. The mitochondrial functions are important in the ROS production associated with aging [47]. The rising lactate and/or PGC-1 $\alpha$ levels and improved mitochondrial functions may help to elevate VEGF levels. The induction of VEGF in the stomach may have contributed to the maintenance of blood flow.

In contrast to the stomach, the sigmoid colon of old rats may be negatively affected by exercise as regards oxidative status. A study conducted on dogs indicated that the regeneration capacity of the large intestine was weaker than that of the small intestine [48]. The weak regeneration capacity of the large 
intestine in this study was linked to the relatively poorer vascular support. An experimental rat study demonstrated that the fractional evaluation of blood flow in different parts of the gastrointestinal tract was 0,4 $\mathrm{mL} /$ minute/gram in the stomach and $0.2 \mathrm{~mL} /$ minute/gram in the rectosigmoid [49]. Another study highlighted the gradual decrease in mucosal capillary density towards the end of the large intestine [50]. Compared to other regions of the gastrointestinal tract, this region -the final section of the large intestine- appears to have a weaker blood supply. In addition, as a result of aging, vascular support gradually worsens. Both in the young and elderly, the effect of exercise on blood flow in this region is unclear in the literature. The increase in the MDA and defensive increase in the intracellular enzymatic antioxidants that were detected in the present study may be related to the vascular sparseness of the sigmoid colon and the substantial reduction in the exercise-related blood flow in old subjects. When compared to the non-exercising group, although the T-AOC level in the sigmoid colon decreased in the exercise group, there was no statistically significant difference. An inverse relationship has been shown in the TNBS-induced colitis model between T-AOC and MDA [51]. We could not detect a decrease in T-AOC levels even though hypoxia related lipid peroxidation increased. Preserving antioxidant capacity in the colon tissues may be the result of triggered mechanisms associated with exercise.

We observed a strong positive correlation between the VEGF and the oxidative markers in the sigmoid colon. This situation indicates that hypoxic influences may be partially effective in triggering VEGF expression. Our findings suggest that hypoxia is more pronounced in the sigmoid colon compared to other proximal parts of the digestive tract. However, the hypoxic effect may not be very harmful due to the preservation of the antioxidant capacity.

In our study with regular mild exercise, we detected no changes in the balance of oxidative status and VEGF levels in the small intestine (both the jejunum and ileum). In a previous study, the fractional blood flow of the small intestine was found to be higher than the other splanchnic regions [52]. In addition, the small intestine has similar ischemia-tolerance mechanisms like the stomach called ischemic preconditioning [53]. A study reported that in the small intestines of rats, repeated intermittent periods of ischemia attenuated intestinal injuries [39]. Stable VEGF and oxidative status in the small intestine may be associated with regular blood flow to this area during exercise.

In this study, the effects of mild exercise on older subjects were examined. The limitation of the study is that compared to the young individuals, changes could not be demonstrated. In the digestive tract the morphological and functional results caused by exercise are also uncertain. Oxidative status and VEGF induction changes in the digestive tract and the clinical consequences of these changes in old subjects can be supported by further studies.

\section{CONCLUSION}

In different sections of the digestive tracts of aged subjects, vascular distribution, adaptation responses, and ischemia tolerance vary, and the effects of exercise on each section is different. It can be speculated that hypoxia does not occur in the proximal part of the gastrointestinal tractus, or adaptive responses against hypoxia are preserved in the stomach, jejunum and ileum tissues of old subjects during regular mild exercise. The stomach was positively affected by mild regular exercise; however, hypoxia was evident in the final section of the large intestine. In this case, regular mild exercise may be recommended to the elderly with complaints for the upper gastrointestinal tract (such as reflux esophagitis, peptic ulcer, gastritis). However, exercise recommendations should be more cautious for the elderly who have complaints concerning the last sections of the digestive tract (such as colonic fibrosis, anal incontinence, rectal polyps and constipation). Although there is a marker of lipid peroxidation in the large intestine, the VEGF induction like the stomach occurs. The VEGF has positive effects on gastrointestinal homeostasis. The cumulative effects of exerciseinduced oxidants/antioxidant and VEGF on the morphology and functions of the digestive tract are open to research.

Funding. This research received no external funding.

Acknowledgments: We would like to thank Joanne Aliye Noonan Kutup who contributed to the English correction of the article.

Conflicts of Interest. The authors declare no conflict of interest. 


\section{REFERENCES}

1. Volianitis S, Secher NH. Cardiovascular control during whole body exercise. J Appl Physiol (1985). 2016 Aug;121(2):376-90.

2. Bilski J, Mazur-Bialy A, Magierowski M, Kwiecien S, Wojcik D, Ptak-Belowska A, et al. Exploiting Significance of Physical Exercise in Prevention of Gastrointestinal Disorders. Curr Pharm Des. 2018 May;24(18):1916-25.

3. Shephard RJ. Peptic Ulcer and Exercise. Sports medicine (Auckland, NZ). 2017 Jan;47(1):33-40.

4. Costa RJS, Snipe RMJ, Kitic CM, Gibson PR. Systematic review: exercise-induced gastrointestinal syndromeimplications for health and intestinal disease. Aliment Pharmacol Ther. 2017Aug;46(3):246-65.

5. Monda V, Villano I, Messina A, Valenzano A, Esposito T, Moscatelli F, et al. Exercise Modifies the Gut Microbiota with Positive Health Effects. Oxid Med Cell Longev. 2017 Mar;2017:3831972.

6. D'Souza AL. Ageing and the gut. Postgrad Med J. 2007 Jan;83(975):44-53.

7. Durazzo M, Campion D, Fagoonee S, Pellicano R. Gastrointestinal tract disorders in the elderly. Minerva Med. 2017 Dec;108(6):575-91.

8. Cardin F, Fratta S, Perissinotto E, Casarrubea G, Inelmen EM, Terranova C, et al. Clinical correlation of mesenteric vascular disease in older patients. Aging Clin Exp Res. 2012 Jun;24(3 Suppl):43-6.

9. Kenney WL, Ho CW. Age alters regional distribution of blood flow during moderate-intensity exercise. J Appl Physiol (1985). 1995 Oct;79(4):1112-9.

10. Apte RS, Chen DS, Ferrara N. VEGF in Signaling and Disease: Beyond Discovery and Development. Cell. 2019 Mar;176(6):1248-64.

11. Schlieve CR, Mojica SG, Holoyda KA, Hou X, Fowler KL, Grikscheit TC. Vascular Endothelial Growth Factor (VEGF) Bioavailability Regulates Angiogenesis and Intestinal Stem and Progenitor Cell Proliferation during Postnatal Small Intestinal Development. PloS one. 2016 Mar;11(3):e0151396-e.

12. Doi A, Kuboki Y, Shitara K, Fukuoka S, Bando H, Okamoto W, et al. Gastrointestinal Perforation and Fistula Formation in 5 Patients With Colorectal Cancer During Treatment With Regorafenib. Clin Colorectal Cancer. 2016 Dec;16(2):E109-13.

13. Sabnis A, Carrasco R, Liu SX, Yan X, Managlia E, Chou PM, et al. Intestinal vascular endothelial growth factor is decreased in necrotizing enterocolitis. Neonatology. 2015 Jan;107(3):191-8.

14. Ahluwalia A, Jones MK, Szabo S, Tarnawski AS. Aging impairs transcriptional regulation of vascular endothelial growth factor in human microvascular endothelial cells: implications for angiogenesis and cell survival. J Physiol Pharmacol. 2014 Apr;65(2):209-15.

15. Guo H, Zhou H, Lu J, Qu Y, Yu D, Tong Y. Vascular endothelial growth factor: an attractive target in the treatment of hypoxic/ischemic brain injury. Neural Regen Res. 2016 Jan;11(1):174-9.

16. Cheng L, Yu H, Yan N, Lai K, Xiang M. Hypoxia-Inducible Factor-1alpha Target Genes Contribute to Retinal Neuroprotection. Front Cell Neurosci. 2017 Feb;11:20.

17. Taylor CT. Hypoxia in the Gut. Cell Mol Gastroenterol Hepatol. 2017 Sep;5(1):61-2.

18. Kuroki M, Voest EE, Amano S, Beerepoot LV, Takashima S, Tolentino M, et al. Reactive oxygen intermediates increase vascular endothelial growth factor expression in vitro and in vivo. J Clin Invest. 1996 Oct;98(7):1667-75.

19. Morland C, Andersson KA, Haugen OP, Hadzic A, Kleppa L, Gille A, et al. Exercise induces cerebral VEGF and angiogenesis via the lactate receptor HCAR1. Nat Commun. 2017 May;8:15557.

20. Vital TM, Stein AM, de Melo Coelho FG, Arantes FJ, Teodorov E, Santos-Galduroz RF. Physical exercise and vascular endothelial growth factor (VEGF) in elderly: A systematic review. Arch Gerontol Geriatr. 2014 SepOct;59(2):234-9.

21. Gavin TP, Robinson CB, Yeager RC, England JA, Nifong LW, Hickner RC. Angiogenic growth factor response to acute systemic exercise in human skeletal muscle. J Appl Physiol (1985). 2004 Jan;96(1):19-24.

22. Jeong J-H, Kim K, Lim D, Kim K-H, Kim H-S, Lee S, et al. Microvasculature remodeling in the mouse lower gut during inflammaging. Sci Rep. 2017 Jan;7:39848.

23. Kawano S, Tsuji S. Role of mucosal blood flow: A conceptional review in gastric mucosal injury and protection. J Gastroenterol Hepatol. 2000 Mar;15(s1):1-6.

24. Magrone $\mathrm{T}$, Jirillo $\mathrm{E}$. The interaction between gut microbiota and age-related changes in immune function and inflammation. Immun Ageing. 2013 Aug;10(1):31.

25. Dumic I, Nordin T, Jecmenica M, Stojkovic Lalosevic M, Milosavljevic T, Milovanovic T. Gastrointestinal Tract Disorders in Older Age. Can J Gastroenterol Hepatol. 2019 Jan;2019:6757524-

26. Tse ACY, Wong TWL, Lee PH. Effect of Low-intensity Exercise on Physical and Cognitive Health in Older Adults: a Systematic Review. Sports Med Open. 2015 Oct;1(1):37-.

27. Arnold JC, Salvatore MF. Getting to compliance in forced exercise in rodents: a critical standard to evaluate exercise impact in aging-related disorders and disease. J Vis Exp. 2014 Aug;(90):51827 
28. Kohler I, Meier R, Busato A, Neiger-Aeschbacher G, Schatzmann U. Is carbon dioxide (CO2) a useful short acting anaesthetic for small laboratory animals? Lab Anim. 1999 Apr;33(2):155-61.

29. Granger DN, Holm L, Kvietys P. The Gastrointestinal Circulation: Physiology and Pathophysiology. Compr Physiol. $2015 \mathrm{Jul} ; 5(3): 1541-83$.

30. Knight KA, Moug SJ, West MA. Systematic review: the impact of exercise on mesenteric blood flow and its implication for preoperative rehabilitation. Tech Coloproctol. 2017 Mar;21(3):185-201.

31. Hearon CM, Jr., Dinenno FA. Regulation of skeletal muscle blood flow during exercise in ageing humans. J Physiol. 2016 Apr;594(8):2261-73.

32. Partovian C, Adnot S, Raffestin B, Louzier V, Levame M, Mavier IM, et al. Adenovirus-mediated lung vascular endothelial growth factor overexpression protects against hypoxic pulmonary hypertension in rats. Am J Respir Cell Mol Biol. 2000 Dec;23(6):762-71.

33. Vilar J, Waeckel L, Bonnin P, Cochain C, Loinard C, Duriez M, et al. Chronic hypoxia-induced angiogenesis normalizes blood pressure in spontaneously hypertensive rats. Circ Res. 2008 Sep;103(7):761-9.

34. Touyz RM, Lang NN, Herrmann J, van den Meiracker AH, Danser AHJ. Recent Advances in Hypertension and Cardiovascular Toxicities With Vascular Endothelial Growth Factor Inhibition. Hypertension. 2017 Aug;70(2):2206.

35. Becejac T, Cesarec V, Drmic D, Hirsl D, Madzarac G, Djakovic Z, et al. An endogeous defensive concept, renewed cytoprotection/adaptive cytoprotection: intra(per)-oral/intraastric strong alcohol in rat. Involvement of pentadecapeptide BPC 157 and nitric oxide system. J Physiol Pharmacol. 2018 Jun;69(3).

36. Mingol-Navarro F, Ballester-Pla N, Jimenez-Rosellon R. Ischaemic conditioning of the stomach previous to esophageal surgery. J Thorac Dis. 2019 Apr;11(Suppl 5):S663-s74.

37. Majumdar AP, Fligiel SE, Jaszewski R. Gastric mucosal injury and repair: effect of aging. Histol Histopathol. 1997 Apr;12(2):491-501.

38. Wang $T$, Leng $Y-F$, Zhang $Y$, Xue X, Kang $Y-Q$, Zhang $Y$. Oxidative stress and hypoxia-induced factor $1 \alpha$ expression in gastric ischemia. World J Gastroenterol. 2011Apr;17(14):1915-22.

39. Jia Z, Lian W, Shi H, Cao C, Han S, Wang K, et al. Ischemic Postconditioning Protects Against Intestinal Ischemia/Reperfusion Injury via the HIF-1alpha/miR-21 Axis. Sci Rep. 2017 Nov;7(1):16190.

40. Mazzola D, Fornari F, Vigano G, Oro T, Costa JA, Bertolin T. Spirulina platensis Enhances the Beneficial Effect of Exercise on Oxidative Stress and the Lipid Profile in Rats. Braz Arch Biol Technol. 2015 Dec;58:961-9.

41. Di Meo S, Reed TT, Venditti P, Victor VM. Role of ROS and RNS Sources in Physiological and Pathological Conditions. Oxid Med Cell Longev. 2016 Jul;2016:1245049.

42. Javanbakht MH, Djalali M, Daneshpazhooh M, Zarei M, Eshraghian MR, Derakhshanian H, et al. Evaluation of antioxidant enzyme activity and antioxidant capacity in patients with newly diagnosed pemphigus vulgaris. Clin Exper Dermatol. 2015 Apr;40(3):313-7.

43. Guan G, Lan S. Implications of Antioxidant Systems in Inflammatory Bowel Disease. Biomed Res Int. 2018 May;2018:1290179-.

44. Rezaie A, Ghorbani F, Eshghtork A, Zamani MJ, Dehghan G, Taghavi B, et al. Alterations in salivary antioxidants, nitric oxide, and transforming growth factor-beta 1 in relation to disease activity in Crohn's disease patients. Ann $\mathrm{N}$ Y Acad Sci. 2006 Dec;1091:110-22.

45. Ohno H, Shirato K, Sakurai T, Ogasawara J, Sumitani Y, Sato S, et al. Effect of exercise on HIF-1 and VEGF signaling. The Journal of Physical Fitness and Sports Medicine. 2012 May;1(1):5-16.

46. Moreira OC, Estebanez B, Martinez-Florez S, de Paz JA, Cuevas MJ, Gonzalez-Gallego J. Mitochondrial Function and Mitophagy in the Elderly: Effects of Exercise. Oxid Med Cell Longev. 2017 Aug;2017:2012798.

47. Sanz A. Mitochondrial reactive oxygen species: Do they extend or shorten animal lifespan? Biochim Biophys Acta. 2016 Aug;1857(8):1116-26.

48. Robinson JWL, Haroud M, Winistörfer B, Mirkovitch V. Recovery of Function and Structure of Dog lleum and Colon Following Two Hours' Acute Ischaemia. Eur J Clin Invest. 1974 Dec;4(6):443-52.

49. Steiner Sheldon H, Mueller Gustave C E. Distribution of Blood Flow in the Digestive Tract of the Rat. Circ Res. 1961 Jan;9(1):99-102.

50. Browning J, Gannon B. Mucosal microvascular organization of the rat colon. Acta Anat (Basel). 1986 Jan;126(2):73-7.

51. Arab HH, Salama SA, Eid AH, Omar HA, Arafa el SA, Maghrabi IA. Camel's milk ameliorates TNBS-induced colitis in rats via downregulation of inflammatory cytokines and oxidative stress. Food Chem Toxicol. 2014 Jul;69:294302.

52. Varga F, Csaky TZ. Changes in the blood supply of the gastrointestinal tract in rats with age. Pflugers Arch. 1976 Jul;364(2):129-33. 
53. Miranda CTCBCd, Fagundes DJ, Miranda Ed, Simões RS, Carbonel AAF, Florencio-Silva R, et al. The role of ischemic preconditioning in the expression of apoptosis-related genes in a rat model of intestinal ischemiareperfusion injury. Acta Cir Bras. 2019 Jun;34(5):e201900501-e.

(C) (1) 2020 by the authors. Submitted for possible open access publication under the terms and
conditions of the Creative Commons Attribution (CC BY NC) license
(https://creativecommons.org/licenses/by-nc/4.0/).

